\title{
ARTIGOS
}

\section{Avaliação de genótipos de algodoeiro para resistência a Verticillium dahliae.}

\author{
Rafael Galbieri ${ }^{1,3^{*}}$, Edivaldo $\mathrm{Cia}^{2}$, Milton Geraldo Fuzatto ${ }^{2}$, Margarida Fumiko Ito ${ }^{2 * *}$, Reginaldo Roberto Lüders ${ }^{2}$, Júlio \\ Isao Kondo ${ }^{2}$
}

${ }^{1}$ Instituto Mato-Grossense do Algodão (IMA); ${ }^{2}$ Instituto Agronômico, Caixa Postal 28, 13001-970, Campinas, SP, cia@ @iac.sp.gov.br.; ${ }^{3}$ Parte da dissertação de Mestrado do primeiro autor, Pós-Graduação em Agricultura Tropical e Subtropical/IAC; *Bolsista FAPESP; **Bolsista produtividade em Pesquisa do CNPq.

Autor para correspondência: Rafael Galbieri.rafaelgalbieri@imamt.com.br

Data de chegada: 05/09/2006. Aceito para publicação em: 11/05/2008

\section{RESUMO}

Galbieri, R.; Cia, E.; Fuzatto, M.G.; Ito, M.F.; Lüders, R.R.; Kondo, J.I. Avaliação de genótipos de algodoeiro para resistência a Verticillium dahliae. Summa Phytopathologica, v.34, n.3, p.211-215, 2008

Genótipos de algodoeiro, compreendendo as principais cultivares em uso no Brasil e linhagens provenientes de diversas entidades, foram avaliadas, em condições de casa de vegetação, quanto à resistência genética à murcha de Verticillium. Com o objetivo de proporcionar condições adequadas para a expressão da resistência, foram desenvolvidos, inicialmente, experimentos para verificar a patogenicidade de diferentes isolados e a concentração mais apropriada do inóculo. A partir desses dados, 25 genótipos foram inoculados, na concentração de $10^{6}$ esporos/
mL, pelo método "dipping", com quatro isolados de V. dahliae. Houve diferenças estatisticamente significativas entre os genótipos com relação à resistência a esse patógeno. Deltaopal, IAC 04/236, IAC 04/259, PR 0136 e Fibermax 966 destacaram-se como mais resistentes e Coodetec 410, Destak, Coodetec 401 e EPAMIG 0316 como mais suscetíveis. A avaliação da doença mostrou-se eficiente tanto considerando sintomas internos quanto externos na planta, verificando-se correlação $r=+$ $0,85^{* *}$ entre os dois métodos de avaliação.

Palavras-chave adicionais: murcha de Verticillium, concentração de inóculo, Gossypium hirsutum

\section{ABSTRACT}

Galbieri, R.; Cia, E.; Fuzatto, M.G.; Ito, M.F.; Lüders, R.R.; Kondo, J.I. Cotton genotypes evaluation for resistance to Verticillium dahliae. Summa Phytopathologica, v.34, n.3, p.211-215, 2008

Cotton genotypes, comprising the main cultivars and some breeding lines available in Brazil, were evaluated under greenhouse conditions for the resistance to Verticillium wilt. In order to establish adequate conditions for resistance expression, experiments examining isolate pathogenicity and the most appropriate inoculum concentration were carried out first. Based on the obtained data, 25 genotypes were then inoculated by the "dipping" method at $10^{6}$ spores $/ \mathrm{mL}$ inoculum concentration and the plants were evaluated by considering both internal and external disease symptoms. Significant differences in resistance level were observed among the genotypes. Deltaopal, IAC 04/236, IAC 04/ 259, PR 0136 and Fibermax 966 were more resistant and Coodetec 401, Distak, Coodetec 410 and EPAMIG 0316 more susceptible. Evaluation method was efficient by observing both internal and external symptoms, and the correlation between them was $\mathrm{r}=+0,85^{* *}$.

Additional keywords: Verticillium wilt, concentration inoculum, Gossypium hirsutum

A nomenclatura do fungo causador da murcha de Verticillium do algodoeiro Gossypium hirsutum L. foi considerada controversa devido à similaridade morfológica entre Verticillium albo-atrum Reinke \& Berth e Verticillium dahliae Kleb. No entanto, estudos verificaram que o agente causal da doença em campo é o fungo Verticillium dahliae (16). O patógeno foi constatado, pela primeira vez, na Virgínia em 1914 (4). No Brasil, V. albo-atrum foi descrito por Krug em 1933 (12), e no Estado de São Paulo, Figueiredo et al. (9) relataram somente $V$. dahliae causando doença nos algodoais. O fungo infecta cerca de 400 espécies de plantas (2), em 40 diferentes famílias (16), quase que exclusivamente dicotiledôneas (14).
Essa doença ocorre em todos os continentes, na maioria dos países que cultivam algodão. Há relatos, na Califórnia, de perdas de até $75 \%$ (16). No Brasil, a doença tem ocorrido com maior freqüência em latossolos roxos, ricos em matéria orgânica, principalmente nos Estado de São Paulo e Paraná (5). Embora esta doença não tenha causado problema sério no Brasil, em estudos recentes, verificou-se que são poucos os genótipos resistentes e observações feitas no ano agrícola de 2007/08 mostram que algumas cultivares utilizadas em plantio apresentam suscetibilidade.

Os sintomas provocados pelo patógeno são caracterizados por clorose irregular nas folhas, seguida eventualmente de necrose, epinastia 
e escurecimento dos vasos do xilema. A intensidade dos sintomas depende de vários fatores, dentre eles, variante do patógeno (17); densidade de inóculo $(15,17)$; temperatura (10); genótipos de algodoeiro $(1,6,11,17)$; interação isolados vs. densidade de inóculo (17); genótipos de algodoeiro vs. densidade de inóculo (13) e temperatura vs. genótipos de algodoeiro (3).

Os sintomas vasculares são indicadores mais seguros para identificação de plantas infectadas pelo patógeno (13), em comparação com os sintomas foliares que estão mais sujeitos a fatores ambientais (8) e ao confundimento com outras doenças, pragas e deficiências nutricionais. Convém assinalar que os sintomas são semelhantes aos causados por murcha de Fusarium, provocada pelo fungo Fusarium oxysporumf. vasinfectum. De acordo com Cia et al. (6), a aplicação de uma escala de notas conforme a intensidade de escurecimento dos vasos das plantas, em estudos de campo, foi o melhor método de avaliação da resistência de cultivares. Também Cia et al. (7) testaram sete cultivares para resistência à doença em condições de campo e verificaram diferenças significativas somente quando foram considerados os sintomas internos. De outra parte, Katsantonis et al. (11) julgaram os dois métodos válidos para quantificar a severidade da doença. No entanto, os sintomas internos não se mostraram correlacionados com a produção, em trabalhos realizados por Pullman $\&$ DeVay (15) e Cia et al. (7), ao passo que, para os externos, essa correlação foi observada.

Em teste de resistência de cultivares de algodoeiro a V. dahliae, Cia et al. (6) avaliaram oito genótipos em condição de campo, por dois anos consecutivos, e, 20 materiais em casa de vegetação, concluindo que houve correlação significativa entre os dados de campo e casa de vegetação. Constatação semelhante foi feita por Balmer et al. (1), que testaram, primeiramente, nove genótipos em campo e, em seguida, quatro em casa de vegetação, mostrando que as duas cultivares mais resistentes e as mais suscetíveis no primeiro experimento, repetiram o desempenho em condições controladas.

Schnathorst \& Mathre (17) indicaram que os sintomas são mais severos em casa de vegetação do que em campo, possivelmente devido a utilização de uma concentração maior do potencial de inóculo, em relação ao de campo, ou porque as plantas tornam-se mais suscetíveis em condições controladas.

De acordo com Wallace \& Batson (18), há dois problemas para desenvolver cultivares resistentes à murcha de Verticillium. Um deles é a baixa disponibilidade de materiais genéticos resistentes à doença $\mathrm{e}$ outro é a dificuldade de realizar estudos da resistência em condições de campo, pois nesta situação não há uma distribuição homogênea do patógeno. Cia et al. (6) assinalaram que os genótipos de algodoeiro ficam mais bem avaliados para resistência a $V$. dahliae quando os estudos são complementados com trabalhos sob condições controladas em casa de vegetação.

O presente trabalho teve como objetivo ajustar a metodologia de inoculação em casa de vegetação e verificar a resistência de genótipos de algodoeiro disponíveis no Brasil ao fungo V. dahliae.

\section{MATERIAL E MÉTODOS}

Experimento 1: Isolados de V.dahliae vs. genótipos de algodoeiro Avaliou-se a resistência de oito genótipos (seis cultivares e duas linhagens) de algodoeiro a seis isolados de Verticillium dahliae Kleb.

O experimento foi realizado em casa de vegetação com os genótipos: Deltaopal, Makina, COODETEC 401, IAC 24, Fibermax 977, PR 0136, STONEVILLE 474 e IAC 02-2190. Os isolados de V. dahliae usados foram: 669-2, 669-3, 669-7, 11998, 12087, 12486 da micoteca do Centro de Fitossanidade/IAC, preservados em água destilada e esterilizada, num ambiente a $25^{\circ} \mathrm{C}$. Para o preparo da suspensão de inóculo, o fungo foi repicado em placas de Petri com meio de cultura BDA e mantido a $26{ }^{\circ} \mathrm{C}$ no escuro, durante sete dias. Em seguida, adicionou-se $15 \mathrm{~mL}$ de água destilada em cada placa, realizando-se posteriormente uma raspagem e ajuste da concentração do inóculo de $10^{6}$ conídios $/ \mathrm{mL}$, com auxílio de câmara de neubauer.

O delineamento foi inteiramente casualizado em esquema fatorial, com três repetições, cada uma delas representada por um vaso (volume de $3 \mathrm{~L}$ ) com duas plantas. A inoculação foi realizada 20 dias após a semeadura pelo método "dipping" (19), pela imersão das raízes por oito minutos na suspensão de inóculo de $V$. dahliae.

A avaliação dos sintomas internos foi feita 60 dias após a inoculação, mediante corte do caule das plantas, atribuindo-se notas de 1 a 5 , conforme a extensão dos vasos escurecidos, sendo nota 1 : planta sem sintomas; nota 2: escurecimento de vasos condutores até 1/4 da distância da base para o ápice da planta; nota 3 : escurecimento de vasos condutores até $2 / 4$ da distância da base para o ápice da planta; nota 4: escurecimento de vasos condutores até 3/4 da distância da base para o ápice da planta; nota 5: vasos condutores escurecidos distribuídos por toda a haste principal da planta. Para análise estatística as notas foram submetidas à transformação $\sqrt{x}$, efetuando-se a comparação de médias dos tratamentos pelo teste de Tukey a $5 \%$.

Experimento 2: Concentração de inóculo de V. dahliae vs. genótipos de algodoeiro

Avaliou-se o comportamento de três cultivares de algodoeiro, escolhidas conforme os resultados apresentados anteriormente, sendo Deltaopal mais resistente e IAC 02-2190 e COODETEC 401 mais suscetível. Essas cultivares foram submetidas à suspensão de conídios com quatro diferentes concentrações de inóculo (C.I.) de V. dahliae C.I.: $10^{2}, 10^{4}, 10^{6}$ e $10^{8}$ esporos $/ \mathrm{mL}$., utilizando-se os mesmos isolados do patógeno assinalados anteriormente.

O experimento foi instalado em casa de vegetação, em delineamento de blocos ao acaso, no esquema fatorial com oito repetições, cada uma delas representada por uma planta.

A inoculação foi realizada pelo método "dipping" (19) em suspensões de inóculo com concentrações específicas de cada tratamento. A avaliação foi feita 60 dias após a inoculação, através dos sintomas internos, conforme descrito no experimento 1 .

Experimento 3: Resistência de genótipos de algodoeiro a $V$. dahliae. Após selecionar os isolados mais agressivos e a concentração de inóculo mais apropriada, foi avaliada a resistência de 25 genótipos de algodoeiro a $V$. dahliae em casa de vegetação. Os materiais utilizados foram: Fundação MT 701, COODETEC 401, COODETEC 409, COODETEC 410, Deltaopal, Deltapenta, STONEVILLE 474, PR 0136, PR 0277, CNPA CO 00-337, CNPA CO 01-54472, Makina, Destak, Fibermax 966, Fibermax 977, EPAMIG 0316, IAC 03-2281, IAC 24, IAC 04-1389, IAC 04/948, IAC 04-1449, IAC 04 H-315, IAC 04/236, IAC 04/259 e IAC 04/244.

$\mathrm{O}$ delineamento foi em blocos ao acaso com quatro repetições, cada uma delas representada por quatro plantas, em um vaso de volume de 5 L. Como substrato, foi utilizada a mistura de areia lavada, terra e esterco curtido na proporção de 15:10:6 respectivamente.

A inoculação foi realizada pelo método "dipping", pela imersão das raízes por oito minutos em suspensão de inóculo na concentração de $10^{6}$ conídios $/ \mathrm{ml}$. O inóculo foi preparado pela mistura de quatro isolados de V. dahliae: 669-2, 669-7, 12087 e 12486, na proporção de $25 \%$ cada, os quais apresentaram, em média, maior agressividade no 
Tabela 1. Notas médias atribuídas aos sintomas internos das plantas de algodoeiro em experimento de avaliação de genótipos inoculados com diferentes isolados de Verticillium dahliae.

\begin{tabular}{|c|c|c|c|c|c|c|c|}
\hline \multirow[t]{2}{*}{ Genótipo } & \multicolumn{3}{|c|}{ Isolados $^{1}$} & \multirow{2}{*}{\multicolumn{2}{|c|}{$669-2$}} & \multirow[b]{2}{*}{ 669-3 } & \multirow[b]{2}{*}{11998} \\
\hline & 12087 & $669-7$ & 12486 & & & & \\
\hline Deltaopal & $1,5 \mathrm{ab}^{2} \mathrm{~A}$ & 1,0 a $\mathrm{A}$ & 1,0 a $\mathrm{A}$ & 1,9 & a A & 1,0 a $\mathrm{A}$ & $1,0 \mathrm{a} A$ \\
\hline PR 0136 & $2,2 \mathrm{ab} A$ & 1,0 a $\mathrm{A}$ & 1,0 a $\mathrm{A}$ & 1,5 & a $\mathrm{A}$ & 1,5 a $\mathrm{A}$ & $1,5 \mathrm{a} A$ \\
\hline Makina & $1,5 \mathrm{ab} \mathrm{A}$ & 1,8 ab $\mathrm{A}$ & 1,0 a $\mathrm{A}$ & 1,0 & a A & 2,9 a $\mathrm{A}$ & $1,0 \mathrm{a} A$ \\
\hline Fibermax 977 & $1,8 \mathrm{ab} \mathrm{A}$ & 1,5 ab A & $2,2 \mathrm{ab} A$ & 1,3 & a A & 1,5 a $\mathrm{A}$ & 1,3 a $\mathrm{A}$ \\
\hline IAC 24 & $2,2 \mathrm{ab} A$ & 1,9 ab A & 1,0 a $\mathrm{A}$ & 1,5 & a A & 1,5 a $\mathrm{A}$ & $1,8 \mathrm{a} \quad \mathrm{A}$ \\
\hline STONEVILLE474 & 1,0 a $\mathrm{A}$ & $2,7 \mathrm{ab} \mathrm{AB}$ & 3,2 b $\mathrm{B}$ & 1,5 & a A & 1,0 a $\mathrm{A}$ & 1,8 a $\mathrm{A}$ \\
\hline IAC $02-2190$ & 3,3 ab $\mathrm{AB}$ & 3,3 ab $\mathrm{AB}$ & 4,7 b $\quad B$ & 2,0 & a $\mathrm{AB}$ & 1,0 a $\mathrm{A}$ & 1,5 a $\mathrm{A}$ \\
\hline COODETEC 401 & 4,0 b $\quad \mathrm{BC}$ & $4,3 \mathrm{~b} \quad \mathrm{C}$ & 1,3 a $\mathrm{AB}$ & 3,6 & a $\mathrm{ABC}$ & 1,8 a $\mathrm{ABC}$ & 1,0 a $\mathrm{A}$ \\
\hline C.V. $(\%)$ & 28,0 & & & & & & \\
\hline
\end{tabular}

${ }^{1}$ Isolados 12087 (Tatuí-SP, 2001); 669-7 (Leme-SP, 1972); 12486 e 11998 (Campinas-SP, 2001); 669-2 e 669-3 (Campinas-SP, 1972).

${ }^{2}$ Médias seguidas na coluna, de letras minúsculas, e na linha de letras maiúsculas, não diferem estatisticamente entre si, pelo teste de Tukey a 5\% de probabilidade.

Tabela 2. Notas médias atribuídas aos sintomas internos na planta em experimento de avaliação de genótipos de algodoeiro em face de diferentes concentrações de inóculo de Verticillium dahliae.

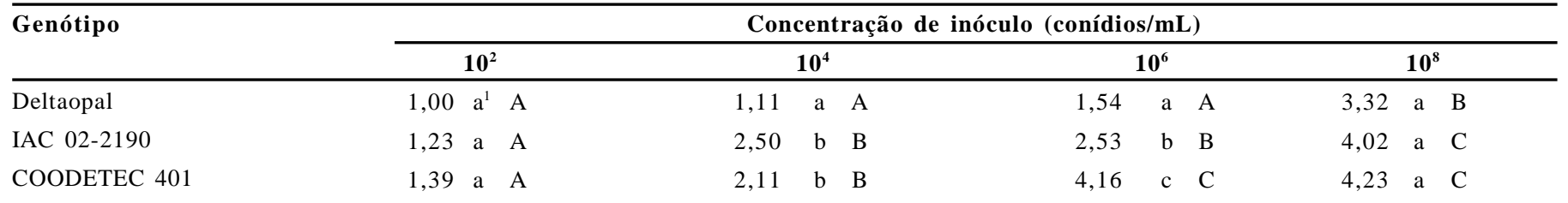

C.V. $(\%)$

11,47

${ }^{1}$ Médias seguidas na coluna, de letras minúsculas, e na linha de letras maiúsculas, não diferem estatisticamente entre si, pelo teste de Tukey a 5\% de probabilidade.

experimento 1 .

A avaliação foi realizada 65 dias após a inoculação através dos sintomas externos e internos. Para os sintomas externos utilizou-se a seguinte escala de notas: 1 : planta sem sintomas; 2 : até $20 \%$ das folhas com sintomas (clorose, com forma irregular, progredindo para necrose); 3: de 21 a $50 \%$ das folhas com sintomas; 4 : de 51 a $80 \%$ das folhas com sintomas; 5 : de 81 a $100 \%$ das folhas com sintomas. A avaliação para sintomas internos foi idêntica àquela apresentada no experimento 1. Para a análise estatística os dados foram transformados em $\sqrt{x}$, efetuando-se a comparação entre as médias pelo teste de Tukey a 5\% de probabilidade. Adicionalmente, foi realizada uma análise de grupamento, utilizando o teste de Scott e Knott a 5\%.

\section{RESULTADOS E DISCUSSÃO}

Experimento 1: Isolados de V.dahliae vs. genótipos de algodoeiro

Houve diferenças significativas entre os genótipos de algodoeiro para resposta à incidência de $V$. dahliae, Tabela 1. Para a maioria dos isolados os genótipos COODETEC 401 e IAC 02-2190 foram os mais suscetíveis e Deltaopal o mais resistente.

Com relação a patogenicidade de V. dahliae, em média, os isolados 12087 e 669-7 foram os mais agressivos, seguidos de 12486 e 669-2. Os isolados 669-3 e 11998 foram os menos agressivos.

Além dos efeitos principais ocorreu significância estatística também para a interação entre genótipos e isolados de $V$. dahliae (F genótipos $\mathrm{x}$ isolados $=1,79 * *)$. Verifica-se que o genótipo STONEVILLE 474 comportou-se entre os mais resistentes aos isolados 669-3 e 12087 e suscetível ao isolado 12486; o genótipo COODETEC
401 foi suscetível aos isolados 669-7 e 12087. O genótipo IAC 022190 mostrou suscetibilidade apenas ao isolado 12486 . Esse fato indica a conveniência de utilizar diferentes isolados para avaliação de resistência de cultivares de algodoeiro a $V$. dahliae. De fato, como relataram Pullman \& DeVay (15), em condições de campo, freqüentemente se observa a existência de populações heterogêneas de V. dahliae, com variação na agressividade das mesmas.

Experimento 2: Concentração de inoculo de V. dahliae vs. genótipos de algodoeiro.

Os resultados obtidos encontram-se na Tabela 2. Confirmando o experimento anterior. $\mathrm{O}$ efeito da interação genótipos x concentração de inoculo foi signifivativo ( $\mathrm{F} \mathrm{G} \mathrm{x} \mathrm{CI}=3,79 * *$ ), também foram encontradas diferenças significativas entre os genótipos de algodoeiro, destacando-se na média, Deltaopal como mais resistente e COODETEC 401 e IAC 02-2190 como mais suscetíveis.

Na média dos genótipos verificou-se efeito linear crescente da concentração de inóculo na intensidade da ocorrência da doença. Esses resultados estão de acordo com os relatados por Schnathorst \& Marthe (17), que testaram concentração máxima de $10^{6}$ e mínima de $10^{2}$ conídios/mL. Contudo, deve-se notar que nas C.I. de $10^{2}$ e $10^{8}$ conídios/ $\mathrm{mL}$ não se verificaram diferenças significativas na resistência das cultivares, ao que tudo indica por insuficiência, num caso, e por excesso, no outro, do patógeno presente. Na C.I. de $10^{4}$ conídios/mL, uma das cultivares mostrou-se significativamente mais resistente do que as outras duas, e na C.I. $10^{6}$ as três cultivares diferiram entre si. Esta última, portanto, mostrou-se a C.I. mais adequada para teste de resistência de genótipo a essa doença. Em síntese, a diferença na 
Tabela 3. Reação de genótipos de algodoeiro a Verticillium dahliae, revelada através de notas atribuídas aos sintomas da doença.

\begin{tabular}{|c|c|c|c|c|c|}
\hline Genótipo & Sintomas & externos & Genótipo & Sintomas & internos \\
\hline Deltaopal & 2,30 & $a^{1}$ & Deltaopal & 3,02 & $\mathrm{a}$ \\
\hline IAC $04 / 236$ & 2,41 & $\mathrm{a}$ & IAC $04 / 244$ & 3,36 & a \\
\hline Fibermax 966 & 2,42 & $\mathrm{a}$ & Deltapenta & 3,38 & a \\
\hline IAC 04-1389 & 2,45 & $\mathrm{a}$ & PR 0136 & 3,38 & a \\
\hline IAC $04 / 259$ & 2,46 & $\mathrm{a}$ & IAC $04 / 259$ & 3,39 & $\mathrm{a}$ \\
\hline CNPA CO 01-54472 & 2,58 & $\mathrm{a}$ & IAC $04 / 236$ & 3,44 & a \\
\hline PR 0136 & 2,65 & a & IAC $04-1449$ & 3,49 & a \\
\hline IAC $04-1449$ & 2,70 & $\mathrm{a}$ & Fundação MT 701 & 3,52 & a \\
\hline IAC 04/H-315 & 2,71 & $\mathrm{a}$ & Fibermax 966 & 3,61 & $\mathrm{a}$ \\
\hline Fundação MT 701 & 2,71 & $\mathrm{a}$ & CNPA CO 01-54472 & 3,64 & a \\
\hline Makina & 2,89 & a & IAC 24 & 3,67 & $\mathrm{a}$ \\
\hline IAC $04 / 244$ & 2,98 & $\mathrm{a}$ & IAC $04 / 948$ & 3,73 & $\mathrm{a}$ \\
\hline Deltapenta & 2,99 & a & IAC 04-1389 & 3,75 & a \\
\hline COODETEC 409 & 3,07 & a & CNPA CO 00-337 & 3,91 & $\mathrm{~b}$ \\
\hline IAC 24 & 3,10 & a & PR 0277 & 3,93 & $\mathrm{~b}$ \\
\hline IAC $03-2281$ & 3,14 & $\mathrm{a}$ & IAC $04 / \mathrm{H}-315$ & 3,95 & $\mathrm{~b}$ \\
\hline IAC $04 / 948$ & 3,18 & $\mathrm{a}$ & Makina & 3,96 & $\mathrm{~b}$ \\
\hline CNPA CO 00-337 & 3,23 & $\mathrm{a}$ & Fibermax 977 & 4,05 & $\mathrm{~b}$ \\
\hline Fibermax 977 & 3,31 & $\mathrm{~b}$ & COODETEC 409 & 4,11 & $\mathrm{~b}$ \\
\hline STONEVILLE 474 & 3,56 & $\mathrm{~b}$ & IAC $03-2281$ & 4,15 & $\mathrm{~b}$ \\
\hline PR 0277 & 3,57 & $\mathrm{~b}$ & STONEVILLE 474 & 4,26 & $\mathrm{~b}$ \\
\hline EPAMIG 0316 & 3,83 & $\mathrm{~b}$ & EPAMIG 0316 & 4,39 & $\mathrm{~b}$ \\
\hline COODETEC 410 & 4,16 & $\mathrm{~b}$ & Destak & 4,45 & $\mathrm{~b}$ \\
\hline Destak & 4,18 & $\mathrm{~b}$ & COODETEC 410 & 4,48 & $\mathrm{~b}$ \\
\hline COODETEC 401 & 4,36 & $\mathrm{~b}$ & COODETEC 401 & 4,53 & $\mathrm{~b}$ \\
\hline Média & 3,07 & & & 3,86 & \\
\hline C.V. & 6,56 & & & 4,90 & \\
\hline $\mathrm{r}^{(2)}$ & +0.85 & $* *$ & & & \\
\hline
\end{tabular}

${ }^{1}$ Teste de Scott e Knott a $5 \%$ de significância.

${ }^{2}$ Correlação entre os sintomas interno e externo

expressão da resistência a $V$. dahliae, em genótipos de algodoeiro, mostrou-se estritamente relacionada com a quantidade de inóculo presente, havendo concentrações mais adequadas para discriminação do material estudado. Nesse aspecto, é útil ressaltar que em concentrações muito altas do patógeno, materiais considerados resistentes podem mostrar reação de suscetibilidade a V. dahliae, como se verificou com a cultivar Deltaopal na concentração de $10^{8}$, no presente trabalho

Experimento 3: Resistência de genótipos de algodoeiro a V. dahliae

Conforme se verifica na Tabela 3, houve diferenças estatisticamente significativas entre os genótipos, considerando tanto os sintomas internos como os externos. Nos dois critérios a cultivar Deltaopal mostrou-se a mais resistente e a COODETEC 401 mostrou-se a de pior desempenho. É interessante assinalar que essas duas cultivares se portaram como extremos, no que diz respeito à resistência, nos três experimentos, mostrando consistência e repetibilidade dos dados. Pelo teste de Scott e Knott pelo menos dois grupos foram estabelecidos. No de melhor desempenho destacaram-se como mais resistentes os genótipos Deltaopal, IAC 04/236, IAC 04/259, PR 0136 e Fibermax 966; no de pior comportamento os genótipos mais suscetíveis foram COODETEC 401, Destak, COODETEC 410 e EPAMIG 0316. É oportuno ressaltar, todavia, o predomínio de genótipos com certo grau de suscetibilidade, mesmo no primeiro dos grupos citados.

Confirmando os resultados obtidos por Katsantonis et al. (11), as duas formas de avaliação da doença, tanto através dos sintomas externos como dos internos, foram equivalentes para a discriminação dos materiais, tendo sido observada correlação de $r=+0.85^{* *}$ entre elas.

\section{AGRADECIMENTOS}

À FAPESP pelo auxílio financeiro à este trabalho.

\section{REFERÊNCIAS BIBLIOGRÁFICAS}

1.Balmer, E.; Cia, E.; Salgado, C.L. Estudo da resistência a Verticillium albo-atrum Reinke \& Berth em variedades paulistas de algodoeiro. Bragantia, Campinas, v. 28, p. 39-46, 1969.

2. Bell, A.A. Verticillium wilt. In: Hillocks, R.J. (Ed.). Cotton disease. C.A.B. London: International, 1992, p. 87-126.

3. Bell, A. A. Diseases of cotton. In: Smith, C. W.; Cothren, J. T. (Ed.). Cotton: origin, history, tecnology and production. John Wiley: New York, NY, 1999, p. 553-593, 1999.

4. Carpenter, C.W. The Verticillium wilt problem. Phytopathology, St. Paul, v. 4, p.393, 1914.

5. Cia, E.; Salgado, C.L. Doenças do algodoeiro (Gossypium spp.). In: Kimati, H.; Amorim, L.; Rezende, J.A.M.; Bergamin Filho, A.; Camargo, L.E.A. (Ed.). Manual de fitopatologia: doenças 
das plantas cultivadas. 4. Ed., São Paulo: Agronômica Ceres, v. 2, 2005, p. 41-52.

6. Cia, E.; Ferraz, C.A.M.; Gridi-Papp, I.L; Silva., N.M.; Soave, J.; Paradela Filho, O.; Ribeiro, I.J.A.; Sabino, N.P. Avaliação da resistência de variedades paulistas de algodoeiro à murcha de Verticillium. Summa Phytopathologica, Botucatu, v. 1, p. 132 $140,1975$.

7. Cia, E.; Ferraz, C.A.M.; Silva, N.M.; Fuzatto, M.G. Comportamento de variedades paulista de algodoeiro em solo infestado pelo fungo causador da murcha verticilar. Bragantia, Campinas, v. 29, p. 73-79, 1970 .

8. DeVay, J.E.; Forrester, L.L.; Garber, R.H.; Butterfield, E.J. Characteristics and concentration of propagules of Verticillium dahliae in air-dried field soils in relation to the prevalence of Verticillium wilt in cotton. Phytopathology, St. Paul, v. 64, p. 22-29, 1974.

9. Figueiredo, M.B.; Teranishi, J.; Cardoso, R.M.G. Estudos sobre espécies do gênero Verticillium parasitas de plantas no Estado de São Paulo. Revista da Sociedade Brasileira de Fitopatologia, Piracicaba, v. 4, p.10-12, 1971.

10. Garber, R.H.; Presley, J.T. Relation of air temperature to development of Verticillium wilt on cotton in the field. Phytopathology, St. Paul, v. 61, n.1, p.204-207, 1971.

11. Katsantonis, D.; Hillocks, R.J.; Gowen, S. Comparative effect of root-knot nematode on severity of Verticillium and Fusarium wilt in cotton. Phytoparasitica, Rehovot, v. 31, n. 2, p. 154-162, 2003 .
12. Krug, H.P. Conhecimento actuaes sobre a murcha do algodoeiro no Estado de São Paulo. Instituto Agronômico de Campinas, Campinas, n.21, 1935. 11p. Boletim Técnico.

13. Paplomatas, E. J.; Bassett, D. M.; Broome, J.C.; DeVay, J.E. Incidence of Verticillium wilt and losses of cotton cultivars (Gossypium hirsutum) based on soil inoculum density of Verticillium dahliae. Phytopathology, St. Paul, v. 82, p. 1417-1420, 1992.

14. Parry, G. Défense des cultures. In: Parry, G. Le cotonnier et ses produits. G.-P. Maisonneuve \& Larose, Paris: 1982. p. 127-180.

15. Pullman, G.S.: DeVay, J.E. Epidemiology of Verticillium wilt of cotton: A relationship between inoculum density and disease progression. Phytopathology, St. Paul, v. 72, n. 5, p. 549-554, 1982.

16. Schnathorst, W.C. Verticillium wilt. In: Watkins, G.M. (Ed). Compendium of cotton disease. American Phytopathological Society, St. Paul: 1981. p. 41-44.

17. Schnathorst, W.C.; Marthre, D.E. Host range and differentiation of a severe form of Verticillium albo-atrum in cotton. Phytopathology, St. Paul, v.56, p. 1155-1161, 1966.

18. Wallace, T.P.; Batson Jr, W.E. Evaluation of obsolete variety collection for resistance to Verticillium wilt. Proceedings of Beltwide Cotton Conference Production. National Cotton Council of America, Memphis v.1, p. 104, 1997.

19. Willes, A.B. A seedling inoculation technique for testing cotton varieties for resistance to Verticillium wilt. Phytopathology, St Paul, v.42, p. 288, (abstr.) 1952. 